\title{
ISÓCRATES Y EL CRÍTICO ANÓNIMO DEL EUTIDEMO DE PLATÓN
}

\author{
Francisco Villar \\ bttps://orcid.org/0000-0001-9713-8943 \\ Universidad de Buenos Aires \\ Consejo Nacional de Investigaciones Científicas y Técnicas \\ bttp://dx.doi.org/10.15304/ag.39.2.6395
}

\section{Resumen}

El presente artículo propone una lectura del Eutidemo de Platón a partir de la escena que tiene lugar en el prólogo del diálogo, en el cual un personaje misterioso critica a Sócrates y a los hermanos erísticos por la conversación que acaba de tener lugar. Defenderé que esta figura anónima esconde a Isócrates, quien en Contra los sofistas y Encomio de Helena había atacado a todos los discípulos de Sócrates por dedicarse a un tipo de actividad intelectual a su juicio volcada a la pura disputa y sin utilidad para la vida cívica. Propondré que el Eutidemo constituye una respuesta a dicha crítica, en tanto que los dos modelos protrépticos de la obra le permiten a Platón distinguir su forma de emplear la dialéctica de la de otros de sus condiscípulos, principalmente los megáricos, así como sentar las bases de su proyecto filosófico-político fundado en el concepto de conocimiento.

Palabras clave: Platón, Isócrates, socráticos, filosofía, erística.

\section{Abstract}

This article proposes an interpretation of Plato's Euthydemus based on the scene that takes place in dialogue's epilogue, in which a mysterious character criticises Socrates and the eristic brothers for the conversation that has just taken place. I will defend that this anonymous figure hides Isocrates, who in Against the Sophists and Encomium of Helen had attacked all the disciples of Socrates for dedicating to a type of activity aimed, in his opinion, at pure contention and without any usefulness for civic life. I will propose that Euthydemus

Recibido: 05/11/2019. Aceptado: 18/02/2020. 
constitutes a response to this criticism because the two protrectic models of the dialogue allow Plato to distinguish his own way of using the Dialectics from that of others of his fellow disciples, mainly the Megarians, as well as to lay the foundations of his philosophicalpolitical project grounded in the concept of knowledge.

Keywords: Plato, Isocrates, Socratics, Philosophy, Eristics.

\section{Introducción}

El Eutidemo constituye un hito fundamental para comprender la respuesta de Platón al interrogante acerca de cuál es el método de la filosofía. El diálogo confronta dos maneras de hacer uso de la dialéctica: de un lado está la erística, llevada a cabo por los hermanos Eutidemo y Dionisodoro; del otro Sócrates, quien en contraposición a estos últimos, ofrecerá otra aproximación a la argumentación dispuesta en preguntas y respuestas. Si el objetivo de los primeros está puesto en la refutación radical de su adversario sin atender ni a los contenidos ni al valor veritativo de lo refutado, y empleando para ello una amplia variedad de recursos engañosos e ilícitos, la tarea del segundo se revelará cooperativa y constructiva: ni el interlocutor será un rival a vencer ni las cuestiones sobre las que versan los argumentos estarán para Sócrates desvinculadas de su consistencia y adecuación a la verdad.

La referencia al método filosófico no es accidental: el concepto de

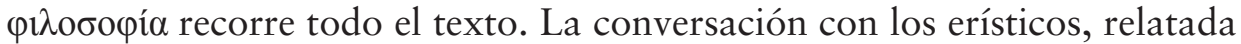
por Sócrates debido a la petición de Critón (271a), tiene lugar porque quie-

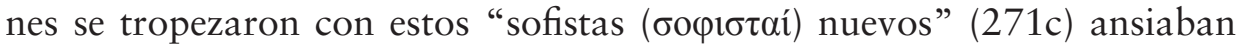
que ambos exhorten a Clinias a la filosofía (

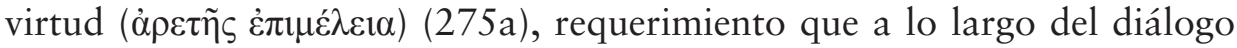
remitirá a la necesidad de educación del muchacho y al deseo de que este se vuelva mejor y lo más sabio posible. Aunque desde la perspectiva de Sócrates los hermanos no cumplirán con tal empresa, algo que dará pie a que él mismo tome a su cargo la exhortación, desde la de sus contrincantes la erística coincide con el ejercicio de la virtud (273d) y constituye su manera de consagrarse a la labor formativa.

El epílogo de la obra constata que es el sentido de la actividad filosófica lo que está en discusión. Critón relata en 304c-305d que una vez disgregada la multitud que rodeaba a los participantes de la conversación, un misterioso personaje anónimo se acercó a él para criticar con virulencia y en su integridad toda la escena que acababa de tener lugar, dirigiendo su invectiva no sólo contra los erísticos, sino también contra Sócrates y cualquiera 
dedicado a la filosofía. El ataque del innominado, como analizaremos en detalle, despertará la reacción de Sócrates, quien a pesar de sus reparos respecto de la práctica discursiva de Eutidemo y Dionisodoro, no dudará en colocarse junto a ellos en lo que respecta a su ocupación en una misma actividad, denominada $\varphi \imath \lambda$ oбopía reiteradamente desde el inicio hasta el final del diálogo.

El presente trabajo se propone brindar una interpretación del Eutidemo atendiendo específicamente a este encuentro final. La lectura está en consonancia con una hipótesis ya defendida por varios especialistas, según la cual tras la figura del crítico anónimo debe ser identificado Isócrates. ${ }^{1} \mathrm{La}$ mayoría de los comentadores del diálogo acuerda en que este personaje representa aquella mirada externa a la dialéctica que no puede vislumbrar las diferencias entre las variantes de ella empleadas por Sócrates y los erísticos, lo cual da por resultado que condene como erística a tal metodología como un todo. Como veremos, no sólo el perfil intelectual del innominado se parece al isocrático, sino que sus ataques a la erística encuentran ecos en Contra los sofistas y Encomio de Helena, discursos en los cuales Isócrates presenta los lineamientos de su propuesta educativa, que él mismo conside-

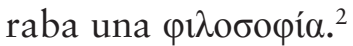

La incorporación de Isócrates permite reforzar otra importante tradición de lectura del Eutidemo, según la cual los hermanos no deben ser considerados sofistas del siglo $\mathrm{V}$ a. C., sino integrantes del círculo liderado por el socrático Euclides de Mégara. ${ }^{3}$ El Contra los sofistas, en efecto, es para

${ }^{1}$ Cf. especialmente Méridier (1931, pp. 25-27), Hawtrey (1981, pp. 133-138), Dusanic (1999) y Mársico e Inverso (2012, pp. 90-93), a los cuales se pueden sumar Canto (1989, pp. 34-36), Dorion (2000, p. 42) y Sermamaglou-Soulmadi (2014, pp. 143-152). Chance (1992, pp. 200-201) considera que el personaje innominado refiere más a un tipo de intelectual, del cual Isócrates sería solo una instanciación.

${ }^{2}$ Para una reconstrucción del pensamiento de Isócrates en tanto filosofía, Cf. Schiappa (1995), Timmerman (1998) y Livigstone (2007). Acerca de la confrontación entre Platón y Isócrates en pos de reapropiarse del término piخoбopía, Cf. Schiappa (1990) y Cole (1991) y Nightingale (1995).

${ }^{3}$ A favor de la interpretación sofística, Cf. Sprague (1972, pp. 294-295), Kerferd (1981, p. 53), Canto (1989, pp. 26-33) y Corey (2015, pp. 121-122). A favor de la megárica, Cf. Méridier (1931, pp. 128-130), Hawtrey (1981, pp. 28-30), Dorion (2000), Mársico e Inverso (2012, pp. 42-58) y Gardella (2013). Hitchcock (2000) apunta a seguidores de Sócrates como origen de la erística, aunque sin sugerir ninguno en particular. Rappe (2000) rastrea los elementos antisténicos a lo largo de toda la obra. En una posición neutral respecto de la identidad de Eutidemo y Dionisodoro se ubican Narcy (1984), Chance (1992) y Sermamoglou-Soulmadi (2014), quienes toman como un dato que ambos son llamados sofistas en Eutidemo 271c. 
numerosos expertos un ataque contra todos los discípulos de Sócrates en el que se los acusa de engañar a sus alumnos y prometer una falsa educación, puesto que su actividad se pierde en argumentos engañosos y paradojas de nula utilidad. ${ }^{4}$ El Eutidemo sería la respuesta de Platón a dicha acusación: aunque similares en apariencia y con un linaje común, la forma de emplear la dialéctica de algunos de sus condiscípulos no debe confundirse con la utilizada por él mismo.

Si bien la hipótesis isocrática ha sido defendida por muchos autores, consideramos que dos defectos en su tratamiento ameritan una revisión. En primer lugar, la tendencia a datar tardíamente el Encomio de Helena de Isócrates ha llevado a no vislumbrar las referencias a este discurso en el Eutidemo, principalmente respecto de la inclusión de las paradojas antisténicas en la segunda demostración erística (283b-288c), mencionadas explícitamente en el Encomio. Incorporar esta obra al análisis permitiría reforzar la idea de que Platón busca tomar distancia de sus compañeros a causa del ataque isocrático. En segundo lugar, los comentadores tienden a concentrarse en el componente metodológico de la respuesta platónica, dejando de lado la réplica al componente doctrinal de la crítica de Isócrates a los socráticos: que su actividad no posee utilidad y, por tanto, no puede servir como fundamento para la vida cívica.

La hipótesis que defenderé será que los modelos protrépticos de Sócrates constituyen no sólo un contrapunto a la actividad de los erísticos, sino también una justificación del lugar de la filosofía en el ámbito de la acción, principalmente a través del nexo que allí se establece entre conocimiento y política. La estrategia platónica del Eutidemo radica en responder doblemente a la crítica de Isócrates: en primer lugar, Platón muestra que su modo de emplear la dialéctica excede el plano de las refutaciones y las paradojas; en segundo, que lejos de no ofrecer a sus discípulos las habilidades necesarias para propiciar la consecución de fines útiles, la filosofía como vía hacia la sabiduría y el conocimiento constituye la única base firme para la política.

El artículo tiene tres partes. En la primera ofrezco un breve recorrido por el ataque de Isócrates a los socráticos en Contra los sofistas y Encomio de Helena, destacando su interpretación erística de la argumentación dialéctica del grupo y el diagnóstico en clave de inutilidad de su propuesta educativa. En la segunda parte reconstruyo en detalle el epílogo del Eutide-

${ }^{4}$ Sobre este punto seguiremos la hipótesis de Mársico (2014, pp. 9-21), quien ha argumentado a favor de un ataque general a los socráticos y no a discípulos en particular, interpretación que permea los trabajos de Jebb (1876, pp. 49-53), Norlin (1929), Van Hook (1945), Mirhady y Too (2000) y Usacheva (2013). 
mo a fin de identificar a Isócrates como el crítico innominado de la filosofía y reconocer los elementos criticados por este en los discursos analizados. En la tercera, finalmente, analizo los dos modelos protrépticos de Sócrates desde una doble perspectiva: por un lado, como respuesta al componente metodológico del ataque isocrático; por otro, como respuesta a su componente doctrinal, en tanto que la posición que Sócrates construye cooperativamente junto con Clinias constituye un modelo de formación que enfatiza la utilidad práctica y política.

Las obras de Isócrates que analizaremos forman parte de los discursos que escribió para promover la filosofía impartida en su escuela, fundada en Atenas a inicios del siglo IV a. C. y en la que ofrecía enseñanza remunerada a la élite local. En el caso de Contra los sofistas, se trataría del primer escrito en el marco de su labor educativa, compuesto en torno al 390 a. C. Aunque está inconcluso, conservamos las secciones destinadas a criticar a otros sofistas, destacando que aquí бoфıбтńs posee un significado genérico cercano a su valor originario como sinónimo de sabio (бoфós). ${ }^{5}$ En cuanto al Encomio de Helena, el discurso posee dos partes bien diferenciadas: un proemio donde otros educadores son atacados (1-15) y el encomio propiamente dicho (16-66). Se lo suele ubicar en el 370 a. C. (Van Hook 1945, p. 59 y Mirhady y Too 2000, p. 32), pero dada su similitud en estilo y propósito con Contra los sofistas y Busiris, preferimos la decisión de Mathieu y Brémond (1962, p. 160) de datarlo a inicios del siglo IV a. C.

El Encomio abre con la primera de las seis críticas que incluye su proemio:

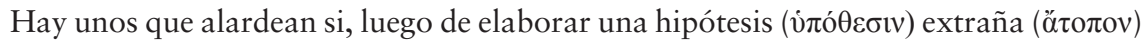
y paradójica ( $\pi \alpha \rho \alpha ́ \delta o \xi o v)$, pueden hablar de ella de modo aceptable. Y se han vuelto viejos; unos afirmando que no es posible decir falsedades, ni contradecir, ni oponer dos discursos sobre las mismas cosas; otros argumentando que la valentía, la sabidu-

${ }^{5}$ En el Eutidemo бoфıбтń posee este significado. Critón indica en 271c: "Son, según pa-

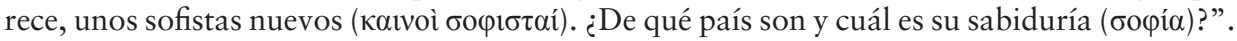
Sobre бoфıбт́́s, Grote (1850, p. 479-485) y Guthrie (1969, p. 38-45) coinciden en: (a) su sinonimia originaria con бoфós; $(b)$ la amplitud de personas a las cuales era aplicado (poetas, músicos, adivinos, legisladores, técnicos, etc.); $(c)$ su carácter elogioso en oposición a la connotación peyorativa que adquirirá durante el siglo IV a. C. Sobre su empleo en Jenofonte, también genérico e inespecífico, Cf. Classen (1984). Respecto del significado que el término adquirirá para Platón en Sofista y para Aristóteles en Refutaciones sofísticas, en ambos casos técnico y peyorativo, Cf. Corey (2015) y Classen (1979) respectivamente. 
ría y la justicia son lo mismo, que no tenemos ninguna de estas por naturaleza, y que

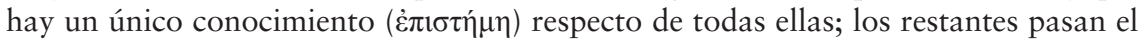

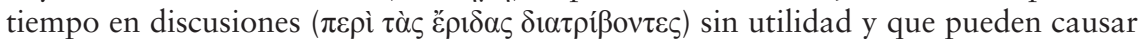
inconvenientes a los que están en su compañía. (Encomio de Helena 1$)^{6}$

La crítica se propone englobar a los atacados en la actividad de elaborar alguna "suposición" o "propuesta" (نં ó $\theta \varepsilon \sigma ı \varsigma)$ que es calificada de ö́o $\pi \alpha \rho \alpha ́ \delta$ o $o_{0}$, vocablos que harían referencia a su carácter inusual y contrario a las creencias comunes. Continúa con una explicitación de ciertas doctrinas y prácticas de las que estos personajes se habrían servido, estableciendo una distinción de tres conjuntos a su interior. A los dos primeros les adscribe una serie de proclamas teóricas que retoman la denuncia de rareza y paradojicidad: al primero tres negativas de orden lógico; al segundo tres afirmaciones éticas que defienden la unidad de ciertas virtudes, el rechazo de su condición natural y su vinculación con un conocimiento único, algo que acentuaría su adquisición no natural. Al tercero lo relaciona con disputas o controversias de las que niega rotundamente su utilidad y tímidamente sugiere su carácter dañino.

Los estudios sobre Isócrates han tendido a compartimentar la crítica (Jebb 1876, p. 51; Van Hook 1945, p. 61, n. a; Mirhady y Too 2000, p. 32 , n. 5): las tesis de los primeros harían referencia a las paradojas sobre la imposibilidad de la falsedad y la contradicción de Antístenes (SSR V.A.147159); ${ }^{7}$ las de los segundos al intelectualismo defendido por Platón en Protágoras 328d-334b y 349a-360e; las disputas de los terceros a Euclides y sus seguidores, los más reputados erísticos (D. L. II.106=SSR II.A.23). Contra esta lectura cabe señalar que no sería correcto circunscribir tales doctrinas y prácticas a estos socráticos en particular: la unidad de la virtud también fue defendida por los megáricos (D. L. VII.161=SSR II.A.32) y hay indicios de intelectualismo en Antístenes (D. L. VI.11-12=SSR V.A.134). Asimismo, todos los discípulos de Sócrates hicieron uso de un patrón argumentativo dialéctico y adoptaron el diálogo como tipo textual, al punto que esto podría considerarse una marca identitaria del grupo. ${ }^{8}$

${ }^{6}$ Me he servido de la edición de Méridier (1931) del Eutidemo y de Norlin (1929) y Van Hook (1945) del Contra los sofistas y el Encomio de Helena isocráticos. Salvo ligeras modificaciones, las traducciones del Eutidemo pertenecen a Mársico e Inverso (2012). Las traducciones de Isócrates me pertenecen.

${ }^{7}$ Las fuente socráticas están referidas a partir de la fuente original y la numeración de Giannantoni (1990)

${ }^{8}$ Para el diálogo socrático como género, Cf. Rossetti (1974), Clay (1994) y Mársico (2010, pp. 40-48). La importancia de la refutación está sólidamente atestiguada en las fuentes sobre el Alcibíades (SSR VI.A.48 y 51) y el Aspasia (SSR VI.A.70) de Esquines. En el 
Una razón interna al discurso también debería alertarnos de los inconvenientes de una división tan férrea. El ataque, en efecto, comienza a partir de un punto en común que solo luego es dividido. Es claro que Isócrates se dirige a un único grupo, algo que se comprueba inmediatamente después: la segunda crítica (2-4) insiste en la falta de originalidad de estos sofistas, los cuales siguen ahondando en las mismas cuestiones inútiles y absurdas de los de la generación anterior, entre quienes Isócrates destaca a Protágoras, Gorgias, Zenón y Meliso. Cabe señalar que los rasgos metodológicos referidos al inicio en tercer lugar se adscriben a todos en la tercera crítica (4-5), dirigida específicamente contra su argumentación: "una pedantería ( $\tau \varepsilon \rho \theta \rho \varepsilon i ́ \alpha)$

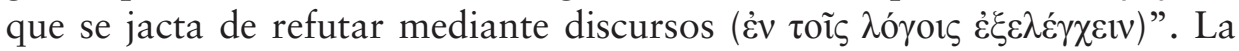
indicación posterior de que es "la filosofía en torno a las discusiones $(\dot{\eta}$

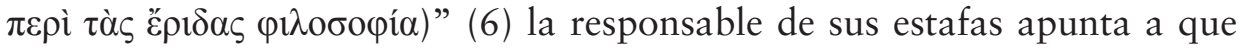
Isócrates no ve discontinuidad entre forma y contenido. El ataque incluye, en efecto, una fuerte afrenta doctrinal, ya que les sugiere que en vez de enseñar tal modalidad discursiva, deberían educar y ejercitar a sus discípulos en las prácticas por las cuales participamos de las instituciones de gobierno, elemento que refuerza con una oposición estructurante de su propuesta: "opinar de modo razonable sobre cosas útiles ( $\pi \varepsilon \rho \grave{~} \tau \tilde{\omega} \nu \chi \rho \eta \sigma i ́ \mu \omega \nu ~ \varepsilon ̇ \pi \iota \varepsilon 1 \kappa \tilde{\omega} \varsigma$ $\delta$ o $\xi \alpha ́ \zeta \varepsilon t v)$ ", que su educación sería capaz de brindar, y "saber con exactitud

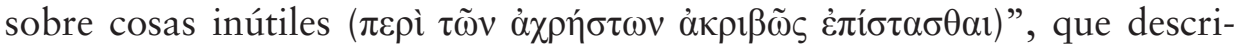
biría la de sus rivales. Isócrates, en síntesis, propone una filosofía anclada en la opinión, la razonabilidad y la utilidad en virtud de enfrentarla a otra basada en el conocimiento, la exactitud y la inutilidad. ${ }^{9}$

El conjunto de rivales de Contra los sofistas incluye tres grupos. El título está en consonancia con que desde el inicio Isócrates se dirige a "los que tienen la intención de educar (oi $\pi \alpha \iota \delta \varepsilon v ́ \varepsilon ı v ~ \varepsilon ̇ \pi \iota \chi \varepsilon i \rho o \tilde{v} \tau \varepsilon \varsigma)$ )" (1). El ataque general contra estos sofistas consiste en indicar que realizan promesas mayores que las que pueden cumplir, algo que ha provocado que las personas comunes tengan en mal concepto a los que se dedican a la filosofía entendida

Banquete de Jenofonte hay referencias a la actitud refutativa de Antístenes (IV.25 y VI.5). El uso del č $\lambda \varepsilon \gamma \chi 0 \varsigma$ está también atestiguado en este socrático. Cf. Económico I y el riguroso análisis de Dorion (2000: CXVII-CLXXXII) sobre el caso jenofonteo.

${ }^{9}$ La cuarta crítica (6) ataca el cobro por enseñanzas inservibles y perjudiciales y podría estar dirigida a Aristipo (D. L. II.54=SSR IV.A.1) y Esquines (D. L. II.83-85=SSR IV.A.144). La última (8-13) reclama que aunque profesen poseer "conocimiento sobre los asuntos políticos ( $\pi \varepsilon \rho \grave{~} \tau \tilde{\omega} v \pi 0 \lambda ı \tau 1 \kappa \tilde{\omega} v \dot{\varepsilon} \pi \iota \sigma \tau \eta ́ \mu \eta v)$ " (9), se atreven a enseñar cosas tales como que la vida de los mendigos y los desterrados es envidiable, posible alusión al elogio de la pobreza de Antístenes (Jenofonte, Banquete IV.34-44=SSR V.A.82) y al modelo de vida errante de Aristipo (Jenofonte, Memorabilia II.1=SSR IV.A.163). 
como actividad educativa. Lo que más preocupa a Isócrates es que las malas acciones de sus competidores socaven su reputación, ya que no todos son capaces de discernir las diferencias. El discurso busca realizar las distinciones necesarias. La presentación del primer grupo ${ }^{10}$ recuerda a los atacados en el Encomio:

En efecto, ¿quién no odiaría y despreciaría, en primer lugar, a los que pasan el tiempo

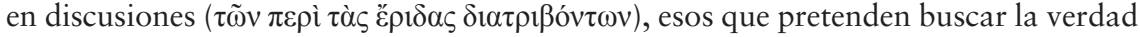

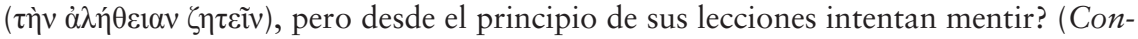
tra los sofistas 1)

A diferencia del primer párrafo del Encomio, donde $\delta \_\alpha \tau i ́ \beta \varepsilon ı v$ se asociaba a êpıs para describir la labor de una parte de los criticados, y que sólo más tarde los englobaba a todos, en Contra los sofistas se hace foco en este elemento desde el principio. El mismo reaparecerá en la última de las cuatro críticas que Isócrates dirige a estos personajes.

La primera (2-3) los vincula con la promesa de poder "conocer de an-

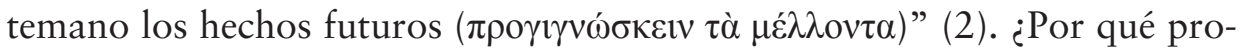
meten este saber? Porque habrían logrado convencer a los jóvenes de que

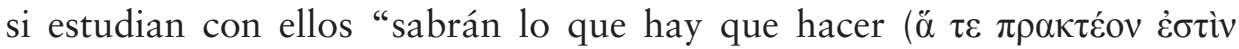

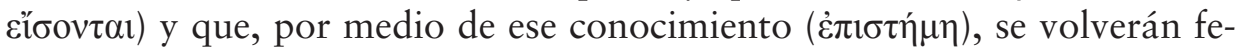

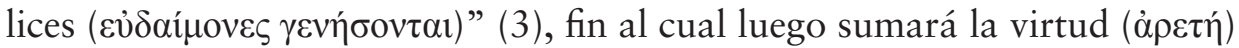

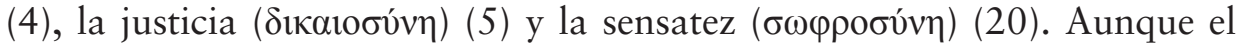
campo semántico en torno a la felicidad no figuraba en el Encomio, este ataque puede ser vinculado con las tesis intelectualistas de su inicio y con el componente doctrinal de la tercera crítica, en donde Isócrates enfrentaba el supuesto saber exacto de sus rivales a su apuesta por la opinión. En lo que se insiste aquí es en considerar estas propuestas robustas como irrealizables y, por lo tanto, engañosas.

La última crítica (7-8) señala que cuando los particulares consideran todos los defectos en conjunto ${ }^{11}$, no llegan a conclusiones distintas a las referidas en el Encomio:

Cuando algunas de las personas comunes, luego de reflexionar sobre todo esto, se dan cuenta de que los que enseñan la sabiduría y transmiten la felicidad carecen de

${ }^{10}$ El segundo grupo (9-18) refiere a logógrafos de promesas exageradas y el tercero (19-20) a sofistas del pasado, prueba de que el primer grupo lo deben integrar intelectuales contemporáneos de Isócrates.

${ }^{11}$ La segunda (3-4) y la tercera crítica (5-6) se dirigen a su modo incongruente de relacionarse con el dinero, como el que pongan poco precio a bienes valiosos como la virtud y la felicidad o que pidan un salario y desdeñen las posesiones. Isócrates no desacredita el cobro en sí mismo, sino que el que este se pida a cambio de enseñanzas inútiles y perjudiciales. 
muchas cosas, y aun así exigen una cantidad pequeña a sus discípulos; de que están atentos a las contradicciones en los discursos, pero no advierten las que se dan en los hechos; de que, además, tienen la pretensión de saber acerca de las cosas del futuro, pero no son capaces de decir ni de aconsejar nada de lo necesario respecto de las del presente; de que están más de acuerdo y más éxito tienen en sus actividades los que se sirven de sus pareceres que los que proclaman tener conocimiento, como era de esperarse, creo, desprecian tales actividades y las consideran charlatanería ( $\dot{\alpha} \delta \mathrm{o} \lambda \varepsilon \sigma \chi i ́ \alpha)$ y argucia argumentativa ( $\mu$ (Sofistas 7-8)

Como ya indiqué, muchos especialistas reconocen a los socráticos en las críticas de ambos discursos. Son pocos, no obstante, los que han destacado la unidad y el carácter integral que estos exhiben respecto del ataque contra este único grupo intelectual, al cual les adjudica dos rasgos principales: $(a)$ una metodología, referida a partir de giros verbales que señalan su gusto por las discusiones, las refutaciones y las contradicciones; $(b)$ un contenido doctrinal, el cual busca fundar nociones éticas, como felicidad y virtud, en propuestas epistemológicas robustas con eje en el conocimiento. Ambos elementos son explícitamente condenados: si a la metodología se la considera inútil y dañina, desde el punto de vista doctrinal se los ataca por ser incapaces de cumplir con sus descomunales aspiraciones y por errar en la determinación de sus medios y fines.

\section{II}

El Eutidemo consiste en un relato (275d-304c) enmarcado por un prólogo (271a-275d) y un epílogo (304c-307c). El prólogo es una conversación entre Sócrates y Critón en la que este último le pide a su amigo que le describa con quiénes dialogó el día anterior en el Liceo, ya que a causa del gentío que los rodeaba no pudo escuchar con claridad. Al parecer de Critón, estos

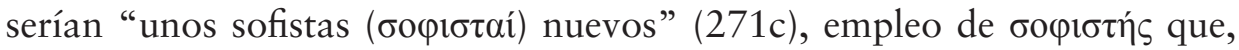
al igual que en Isócrates, posee un sentido genérico referido a su cualidad de sabios (бофоí). La narración de Sócrates es un intento de rememorar

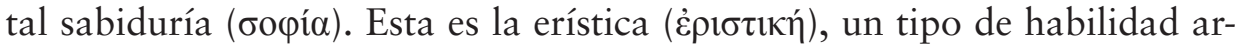
gumentativa que se jacta de refutar cualquier cosa que diga un adversario sin importar su verdad o falsedad. El término épıs remite al ámbito de la "discordia", la "disputa" y la "pelea", aspecto combativo de la refutación erística que sería, en verdad, el despliegue verbal de una capacidad más amplia. Los hermanos, igualmente, se desentienden de su ocupación actual en otros tipos de lucha (física, armada o judicial), indicando que ahora sólo se abocan a la refutación, vinculada por ellos con la transmisión de la virtud. 
La promesa despierta la sorpresa de Sócrates, quien los invita a hacer una

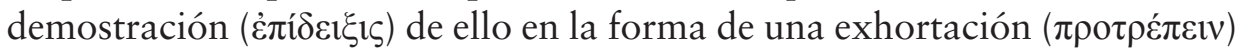
a la filosofía.

Es a partir de este requerimiento, que los erísticos afirman ser capaces de cumplir, que se da inicio a los cinco episodios del diálogo propiamente dicho. De estas secciones, tres tienen como protagonistas a los erísticos (275d-278e, 283b-288c y 293b-304c) y dos a Sócrates (278e-283b y $288 \mathrm{~d}-$ 293a), las cuales se presentan de manera intercalada. Dicha estructura se debe a que a cada intento de los hermanos de brindar una demostración de su sabiduría, Sócrates considerará que el objetivo no ha sido cumplido, suministrando él mismo, en contrapartida, un modelo de cómo debe realizarse un protréptico a la filosofía y la virtud. Tal contraste es lo que le permitirá a Platón elaborar lo que constituye el núcleo del diálogo: contraponer dos prácticas que, aunque similares en apariencia, poseen fundamentos, métodos y fines diferentes.

Una vez finalizado el relato de Sócrates, que culmina con una serie de consejos a los hermanos (303c-304c) respecto de mantener su actividad entre unos pocos alumnos pagos para preservar su valor, ya que la rápida conversión de Ctesipo a la erística en 300d demostró que esta es fácil de imitar, el diálogo retoma la conversación con Critón. ${ }^{12}$ Coherente con la presentación de ambos como expertos en su materia, Sócrates propone que se vuelvan discípulos de Eutidemo y Dionisodoro. Sin embargo, Critón no aceptará la invitación, argumentando que preferiría estar entre los refutados por el tipo de argumentos erísticos que entre los refutadores, punto que alude a una de las advertencias socráticas respecto de la opinión que la mayoría tiene sobre la práctica (303d). Y si bien Critón aclara que le parece ridículo aconsejar a Sócrates, procederá a informarle de un encuentro que tuvo tras culminar el intercambio con los hermanos.

Cuando la multitud se estaba dispersando, se acercó a Critón un hombre del cual este resalta dos características: es considerado muy sabio y es alguien versado en discursos judiciales. El diálogo con el personaje es sugerente, ya que ataca con agresividad a Eutidemo y Dionisodoro. Primero se refiere a ellos como dos sabios dignos de escuchar, indicación que luego se revelará irónica, porque, en rigor, para él no son sabios en términos generales, sino los más sabios entre aquellos que en la actualidad se dedican a este

${ }^{12}$ En 273d Eutidemo afirma: "Creemos ser capaces de conferir la virtud mejor que cualquiera y del modo más rápido". La transformación de Ctesipo en un erístico constituye el éxito de tal promesa: dado que para los hermanos la erística es la virtud, un breve encuentro con Ctesipo ha bastado para transmitírsela. 
tipo de argumentos. A juicio del innominado, estos son "lo que se puede escuchar de esta clase de charlatanes ( $\lambda \eta \rho 0 v \tilde{v} \tau \varepsilon \varsigma)$ y de aquellos que hacen

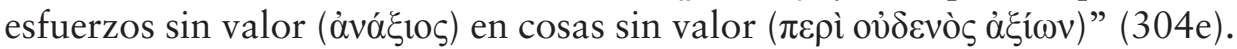

La reacción de Critón será vincular la conversación central del diálo-

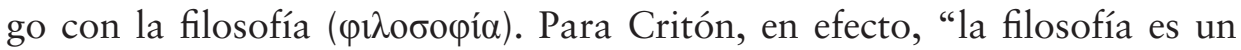

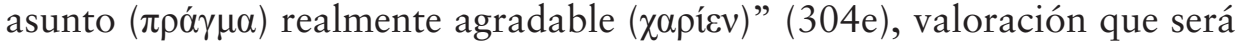
inmediatamente desestimada por su interlocutor, quien se referirá a la filo-

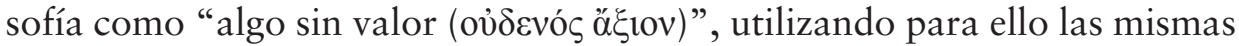
palabras que antes. Lejos de circunscribir la crítica a los erísticos, también arremeterá contra Sócrates, puesto que para este Critón debería avergonzarse de su amigo, el cual, a su juicio, se comportó de un modo "absurdo

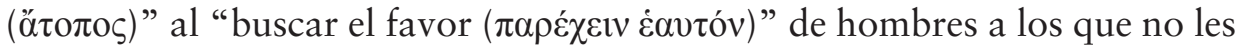
preocupa lo que otros digan, sino que "se dirigen contra cualquier expresión ( $\pi \alpha v \tau o ̀ \varsigma \rho j \eta ́ \mu \alpha \tau o \varsigma ~ \alpha ̉ v \tau \varepsilon ́ \chi \varepsilon \sigma \theta \alpha)$ " (305a), acusación que podría remitir a su imperativo refutativo sin límites. Aunque Critón considera que la censura a la filosofía quizás no es adecuada, acuerda en el reproche a Sócrates. Este era el consejo que temía darle a su amigo. ${ }^{13}$

La invectiva contra la filosofía tomará un cariz personal para Sócrates, quien luego de indagar sobre el perfil intelectual del innominado, procederá a responder a su ataque. En cuanto a los datos sobre el personaje, Critón insistirá en su maestría en asuntos judiciales, aunque dejará sentado que no es un orador — nunca piso un tribunal-, sino un logógrafo. Sócrates indicará que tal tipo de hombres son "el límite ( $\mu \varepsilon \theta$ óptos) entre el filósofo y el político" (305c), dado que dicen dedicarse medidamente ( $\left.\mu \varepsilon \tau \operatorname{cí}^{\prime} \omega \varsigma\right)$ a la filosofía y medidamente a las cuestiones políticas, medianía que, a los ojos de la mayoría, los hace tener el aspecto de ser los más sabios. Es esta búsqueda de fama ( $\left.\delta \delta^{\xi} \xi \alpha\right)$ lo que para Sócrates los guía, y es por ella que quieren desprestigiar a los dedicados a la filosofía: si logran rebajarlos, parecerán más sabios ante el público.

${ }^{13}$ Varios especialistas (Méridier 1931, pp. 133-134; Chance 1992, p. 206; Mársico e Inverso 2012, pp. 90-93) creen que el ataque del innominado se dirige en igual medida a Sócrates y a los erísticos. Aunque esta interpretación podría afianzar la lectura megárica del Eutidemo, ya que como parte del mismo círculo intelectual sería esperable que alguien externo amalgamara a Sócrates con sus discípulos, la misma podría matizarse: a partir de que el innominado critique a Sócrates por intentar "ganarse el favor" o "entregarse" a los hermanos - $\pi \alpha \rho \dot{\varepsilon} \chi \varepsilon ı v \dot{\varepsilon} \alpha v \tau o ́ v-$, quizás pueda extraerse que ve cierta distancia entre ellos. La crítica a Sócrates se da, igualmente, en el marco del reconocimiento general de que este y los hermanos erísticos se ocupan de algo llamado filosofía, y es contra esta que el innominado dirige todas sus diatribas. 
Sócrates tomará este carácter intermedio para elaborar una objeción al innominado en 306a-d. El argumento es el siguiente: toda actividad que se encuentre en medio de otras dos y participe de ambas, las cuales, por su parte, sean buenas y útiles respecto de su objeto específico, dará por resultado algo que será peor que el de las dos prácticas consideras en sí mismas. Dado que la filosofía y la política son buenas, quien se encuentre en medio de ellas y pretenda participar de ambas, estará en peor posición respecto del bien que si se ocupara enteramente a ellas. Por tanto, lejos de ser una ventaja, el carácter pretendidamente medido del cual se jactan personajes como el anónimo es una desventaja en relación con la filosofía, actividad de la que, cabe aclarar, Sócrates considera partícipes no solo a él mismo sino también a los dos erísticos.

Esto último despertará la desazón de Critón, quien compartirá a Sócrates su preocupación acerca de qué hacer con su hijo Critóbulo, que a su juicio ya tiene edad para que alguien lo asista en su formación. Critón desearía que el joven sea orientado hacia la filosofía, pero al juzgar a los que afirman ser capaces de educar a los hombres y constatar su total incapacidad, no sabe cómo proceder. La recomendación final de Sócrates será que se concentre en la actividad misma y no en quienes dicen profesarla, ya que a la filosofía no le ocurriría algo distinto de lo que sucede con cualquier otra ocupación, en donde hay muchos mediocres y los valiosos son pocos. Sólo mediante un examen exhaustivo podría revelarse su verdadero valor, investigación a la cual Critón es finalmente exhortado y que da por concluido el diálogo.

El epílogo del Eutidemo ha suscitado tradicionalmente dos preguntas: cuál es la identidad del innominado y qué sentido tiene su aparición. Una primera opción, como la que defiende Chance (1992, pp. 200-201), es considerar que este personaje no pretende describir a nadie en particular, sino a un tipo de intelectual. Para esta posición importa más su función en el diálogo que su identificación, la cual radicaría en el llamado de atención respecto de ciertas miradas externas que no sobrepasan la similitud aparente entre Sócrates y los erísticos y condenan a la dialéctica en tanto método filosófico como un todo. Bajo esta lectura, la exhortación final a Critón podría leerse como una invitación a superar los ataques superficiales a la filosofía por la vía de la crítica a sus cultores, sin problematizar sobre sus fundamentos, métodos y fines. 
Otra opción que enriquece esta propuesta es incorporar la figura de Isócrates. ${ }^{14}$ Su perfil intelectual, en efecto, se ajusta en varios puntos al del innominado. A partir de sus biografías ${ }^{15}$, por ejemplo, sabemos que tuvo una breve actividad como logógrafo, labor a la cual se habría dedicado por la ruina de su familia durante la guerra del Peloponeso. ${ }^{16} \mathrm{~A}$ pesar de que conservamos algunos de esos trabajos, Isócrates repetidamente criticará la logografía y negará haberse dedicado a ella (Panegírico 188 y Antídosis 36). La insistencia en que el crítico nunca pisó un tribunal y, sin embargo, redacta discursos, estaría también en consonancia con la noticia sobre la voz endeble y el carácter tímido que hicieron rehuir a Isócrates de la política, prefiriendo dejar por escrito sus ideas en discursos que simulaban estar compuestos para su pronunciación. El que en ellos intentara influir a figuras destacadas y aconsejar sobre asuntos públicos lo coloca en el marco de la vía intermedia entre filosofía y política atacada.

La vinculación del innominado con el concepto de $\delta o ́ \xi \alpha$ podría estar relacionada con el valor dado por Isócrates al concepto. En efecto, la propuesta isocrática está más enfocada en brindar una formación que fomente

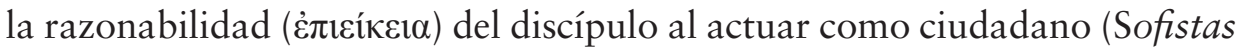
21) que un conocimiento exacto que sea inútil para el ámbito público. La oposición $\delta$ ó $\xi \alpha-\varepsilon \dot{\pi} \iota \sigma \tau \eta \dot{\mu \eta}$ es estructurante de sus discursos y refleja sus aspiraciones mesuradas: su apuesta radica en promover la habilidad para producir y decir discursos políticos, que serían "los que se ocupan de la vida en

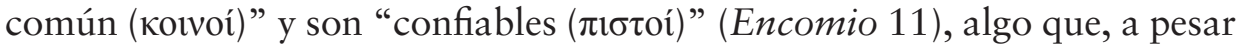
de ser un fin modesto, reconoce que no es fácil de lograr: por un lado, estos deben ser confeccionados y pronunciados atendiendo a una gran variedad

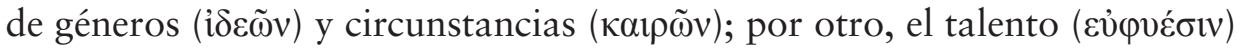
y la experiencia ( $\left.\dot{\varepsilon} \mu \pi \varepsilon \uparrow \rho^{\prime} \alpha\right)$ previa del alumno imponen límites a las posibilidades del maestro (Sofistas 14).

De acuerdo con la lectura de Sócrates de la posición del innominado, es comprensible que alguien enfocado en la utilidad de la palabra en el marco de instituciones públicas, como los tribunales, la asamblea o el Consejo,

\footnotetext{
${ }^{14}$ Remitimos a la nota 1 para un estado de la cuestión sobre Isócrates y su identificación como el crítico.

${ }^{15}$ Contamos con tres vidas de Isócrates: Sobre Isócrates de Dionisio de Halicarnaso (s. I a. C.); Las vidas de los diez oradores del Pseudo-Plutarco (s. II d. C.); y una biografía atribuida a Zósimo (s. V d. C.).

${ }^{16}$ La estancia de Isócrates en Quíos — patria de Eutidemo y Dionisodoro- transmitida por el Pseudo-Plutarco (837B) ha hecho pensar a Dunasic (1999) en una posible referencia a Isócrates en el Eutidemo.
} 
busque censurar a quienes, como Sócrates y los erísticos, se dirijan ante todo a la precisión lingüística y argumentativa en discusiones privadas. A

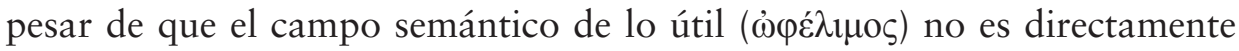
empleado en la escena final del Eutidemo, la negativa a conferir valía a la filosofía encarnada por los participantes de la discusión a través del señalamiento de su falta de ö $\xi ı v$, adjetivo que indica lo que es "de valor", "digno de aprecio", "estimable", etc., acerca poderosamente al innominado a la crítica de inutilidad de Isócrates a los socráticos en Contra los sofistas y Encomio de Helena.

Al igual que en ambos discursos, además, el crítico no realiza una distinción entre la forma y el contenido de lo que en el diálogo es llamado $\varphi \iota \lambda$ produce su falta de valor. Veremos que la estrategia platónica para responder al innominado y, por tanto, a Isócrates, radicará en mostrar cómo una aproximación distinta a la dialéctica podría constituir una base firme para dar con los contenidos útiles que este requiere. Platón acordaría con su diagnóstico de que la erística no es provechosa, idea expresada en la fórmula de que los hermanos "cosen la boca de los hombres" (303e), ya que en el marco de una discusión erística no hay lugar para construcciones positivas. No obstante, el diálogo alerta respecto de que la cercanía aparente entre Sócrates y los erísticos y el que digan ocuparse de la filosofía no debería llevar a un amalgamiento de sus prácticas.

\section{III}

La erística que Eutidemo y Dionisodoro despliegan en las tres demostraciones del Eutidemo posee cuatro rasgos principales. En primer lugar, los hermanos afirman ser capaces de refutar siempre lo que otro diga. Por refutación ( $\check{\lambda} \lambda \varepsilon \gamma \chi 0 \varsigma)$ se debe entender la construcción de un razonamiento que, dada una posición con la cual se ha comprometido su adversario, o que se presume que este acepta ${ }^{17}$, concluye algo incompatible con ella. En segundo lugar, su labor es interrogativa, lo cual implica que se limitan a preguntar y extraer premisas que luego serán usadas para hacerle admitir al rival la refutación. En tercer lugar, a este no le están permitidas ni las repreguntas ni los pedidos de aclaración, así como ningún añadido a la respuesta que

${ }^{17}$ Las primeras serían las refutaciones que Hitchcock (2000, pp. 64-67) llama directas, las segundas las indirectas. En estas últimas alcanza con derivar un absurdo inaceptable de las palabras del interlocutor. Para una reconstrucción detallada de las características de la argumentación erística, Cf. Gardella (2013). 
exceda el "sí" o el "no". Esta regla se explica porque, finalmente, sus argumentos están atravesados por equívocos, ambigüedades e imprecisiones que habilitan la malinterpretación en pos de la refutación, concebida esta como una victoria argumentativa que no busca establecer la verdad de lo concluido, sino sumir al adversario en la perplejidad y el ridículo.

La segunda demostración (283b-288c) incluye, por ejemplo, cuatro argumentos que siguen el hilo conductor de las tesis antisténicas sobre la imposibilidad de la falsedad y la contradicción. Esto no significa que Eutidemo y Dionisodoro abracen tales doctrinas y procuren defenderlas a la luz de las ideas de Antístenes. Estas son empleadas sólo como vehículo para refutar las posiciones que va adoptando Ctesipo: que Dionisodoro miente al decir que quiere destruir a Clinias; que es posible decir cosas que no son; que Ctesipo y Dionisodoro se contradicen al afirmar el uno y el otro que la falsedad es posible e imposible respectivamente. Lo que guía a los hermanos es la problemación de la noción del discurso ( $\lambda$ ó ${ }^{\circ} \varsigma$ ) como aquello que dice lo que es ( $\tau$ ò öv) y, por tanto, la verdad ( $\dot{\alpha} \lambda \hat{\eta} \theta \varepsilon ı 1 \alpha)$, dejando a la falsedad

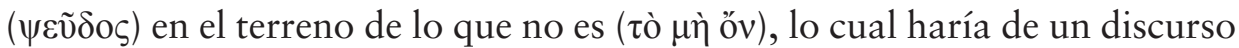
falso algo imposible. ${ }^{18}$

La inclusión de los argumentos de Antístenes y su crítica podría responder a que estos aparecen en el Encomio de Helena. No sería extraño que el Eutidemo también estuviera dirigido a este socrático, quizás no por dedicarse directamente a la erística, como los megáricos, sino por abogar por posiciones contraintuitivas que en manos de personajes como estos podrían ocasionar el descrédito de otros de sus condiscípulos. La posición de Platón respecto de estas paradojas no es distinta a la de Isócrates. La replica a ellas, en efecto, recurre a los problemas pragmáticos que implica su empleo y aceptación: ninguna puede ser sostenida en una conversación sin que su defensor caiga en algún tipo de inconsistencia, principalmente la que se da entre que el objetivo de la refutación es mostrar que el adversario está en el error y la supuesta inexistencia de tal estado de cosas según la tesis. Como sostendrá Sócrates en 286c, el argumento de la inexistencia de la falsedad no sólo anula a los demás, sino también a sí mismo. ${ }^{19}$

${ }^{18}$ Sobre el problema de lo falso en Platón, Cf. Crombie (1963, pp. 486-498) y Denyer (1991). Para un análisis específico de estos argumentos del Eutidemo y su vínculo con el Sofista, Cf. Marcos (2000).

${ }^{19}$ Sócrates presenta al menos otras dos inconsistencias prácticas entre la actitud de los hermanos y su defensa de la ausencia de falsedad y, por tanto, de error e ignorancia: que Dionisodoro le pida que lo refute (286e) y que se hayan presentado a sí mismos como maestros al inicio de la conversación (287a). 
Pero la actitud de Platón frente a los erísticos no radica solo en mostrar sus vicios, sino también en ofrecer una alternativa. Los dos modelos protrépticos de Sócrates presentan otra forma de argumentación que, aunque es dialéctica, no puede igualarse a la erística. Su objetivo no es la refutación, sino construir una posición junto con el interlocutor y no contra él, proceso que se realiza a partir sus aceptaciones y rechazos, acuerdos y desacuerdos. Es posible y deseable que el interrogado adopte un rol activo y coopere en el avance del argumento. ${ }^{20}$ Es gracias a este tipo de intercambio que Sócrates y Clinias son capaces de arribar a conclusiones respecto de la necesidad de volverse más sabios y desarrollar el conocimiento, búsqueda que si bien, veremos, desemboca en una aporía, es más fructífera que la labor negativa de la erística.

A este elemento cooperativo debe sumarse otro rasgo que diferencia a la dialéctica del Eutidemo de la erística: la coherencia argumental. En efecto, a pesar de que Sócrates y Clinias dialogan en dos momentos distintos, en ambos construyen un único razonamiento, en tanto que la segunda demostración retoma exactamente la conclusión de la primera (282d y $288 \mathrm{~d}$ ). Esto confronta con la fragmentación de la indagación de los hermanos, en la cual las refutaciones se suceden con el único fin de perturbar cada vez más al adversario. La consistencia entre los planteos de los dos protrépticos permite a Platón sortear cualquier crítica respecto de la falta de contenido de su propuesta. En lo que resta analizaremos cómo el argumento elaborado en estos podría también constituir una respuesta al desafío doctrinal lanzado por Isócrates.

Lo primero (278d-279c) que Sócrates y Clinias establecen es que los hombres desean que las cosas "le salgan bien (عũ $\pi \rho \alpha ́ \tau \tau \varepsilon เ v)$ ", expresión ambigua que conjuga la idea de "actuar bien" e "irle a uno bien", lo cual

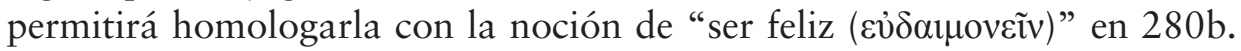
Alcanzaríamos esta aspiración poseyendo muchos bienes ( $\alpha \gamma \alpha \theta \alpha \dot{\alpha})$, entre los que destacan primero el ser rico, estar sano o tener un buen linaje y luego añaden el ser moderado, justo y valiente. A estas virtudes intentarán agre-

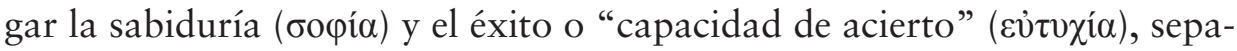
ración que, no obstante, será evaluada como un paso en falso, ya que para Sócrates la sabiduría conduce al éxito en cualquier actividad (279d-280b): sólo el flautista sabio tiene capacidad de acierto respecto de la práctica de

${ }^{20}$ Para un análisis del Eutidemo bajo esta hipótesis de confrontación entre el carácter cooperativo y comprometido con la verdad con los fines combativos y polemistas, Cf. Gill (2000) y Vigo (2001). 
la flauta, y lo mismo ocurre con el piloto respecto de la navegación y con el general respecto de la dirección del ejército.

Determinado esto, en $280 \mathrm{c}-281 \mathrm{~b}$ se argumentará que el modo por el cual los bienes se vuelven útiles no es meramente poseyéndolos, sino usándolos, y no de cualquier manera, sino rectamente (ỏ $\rho \theta \tilde{\omega} \varsigma)$, siendo para ello necesario un conocimiento ( $\left.\dot{\varepsilon} \pi \_\tau \eta \dot{\mu \eta}\right)$ que guíe a la acción. Esto los hará reconsiderar que las cosas que antes llamaron bienes lo sean en y por sí mismas. Tal atribución, por el contrario, depende del principio que asiste al uso (281d):

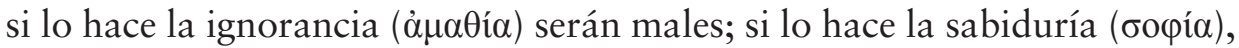
bienes. ${ }^{21}$ Sabiduría e ignorancia son propiamente el bien y el mal, así que para lograr la felicidad y que las cosas nos salgan bien hay que procurar ser lo más sabio posible. Bajo el supuesto de que esto es enseñable, cuestión que Sócrates y Clinias aceptan sin someterla a discusión, sería necesario ir a la búsqueda de enamorados, amigos o tutores que nos asistan en la tarea de volvernos sabios.

La suficiencia de la sabiduría para la consecución de la virtud y la felicidad recuerda las tesis intelectualistas criticadas por Isócrates. En Contra los sofistas 2-3 la primera invectiva a los socráticos radicaba en su promesa

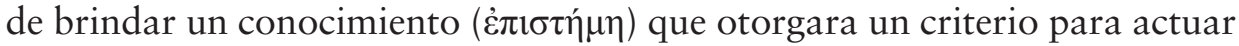
y así lograr la felicidad, la virtud, la justicia y la sensatez. En Encomio de Helena 1, por su parte, al segundo subgrupo dentro de los atacados se le adjudicaba tres posiciones: homologar sabiduría, valentía y justicia, postular que ninguna se da por naturaleza, y afirmar que hay un único conocimiento respecto de todas ellas. Los paralelos no son totales pero sí llamativos en la

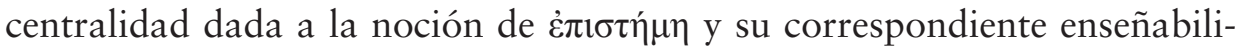
dad, punto que, vimos, constituía para Isócrates el mayor atrevimiento de sus contrincantes.

En el Eutidemo, no obstante, Platón parece hacerse cargo de las bases teóricas a las que se dirige esta acusación, e incluso las profundiza, principalmente a partir del vínculo que el segundo protréptico trazará entre conocimiento y política. Sócrates y Clinias retoman su investigación desde donde la habían dejado antes, recordando en 288c que, por un lado, "se

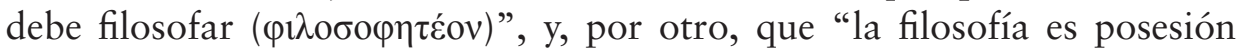

${ }^{21} \mathrm{El}$ argumento es ligeramente más complejo en este punto. Suponiendo que a cada bien antes considerado, como el ser rico, se le opone un contrario, como el ser pobre, quien utilice su riqueza con ignorancia logrará males mayores que quien no tiene esta disponible, ya que así colocaría más poder a cargo de un guía malo. Si a la riqueza la guía la sabiduría sus bienes son mayores. Seguimos la reconstrucción del argumento de Mársico e Inverso (2012, ad. loc.) y Chance (1992, pp. 55-78). 


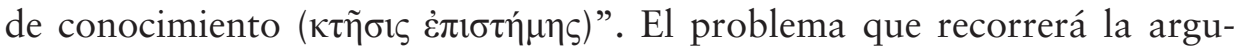
mentación de aquí en más será cuál es dicho conocimiento. Siguiendo los lineamientos de la primera parte de la conversación, Sócrates introduce la condición de que este debe aunar el hacer con el saber usar eso que hace, ya que sólo así estaríamos ante un conocimiento útil y valioso para quien lo posee en vistas a su felicidad.

El segundo paso (289b-290d) consiste en evaluar candidatos en base al principio establecido. En artes como la fabricación de flautas y liras el conocimiento de su producción es obviamente distinto al de su empleo. Lo

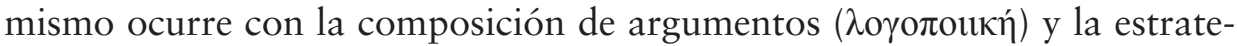

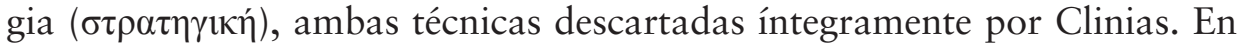
el caso de esta última, el muchacho argumenta que, en tanto caza de hombres, la estrategia sólo sirve para subyugar aquello que apresa, que luego debe entregar a quien sepa usarlo, tal como los pescadores dan sus presas a los cocineros y los matemáticos sus descubrimientos a los dialécticos. La estrategia, en concreto, otorga ciudades y ejércitos a los políticos, ya que ella no sabe emplearlos. Este argumento abre paso a la política y funciona como una muestra de los poderes protrépticos de la conversación con Sócrates, al punto que la intervención de Clinias amerita una ruptura del relato principal para que Critón elogie los avances del joven.

La propuesta superadora es el arte del reinado ( $\beta \alpha \sigma i \lambda \iota \kappa \eta ́)$, respecto de la

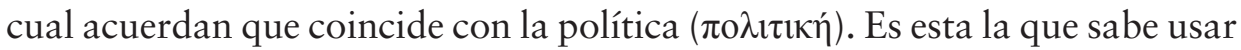
las cosas y hace que se actúe bien en una ciudad, "dirigiendo ( $\kappa \nu \beta \varepsilon \rho v \tilde{\alpha} v)$ y gobernando (ä $\chi \chi \varepsilon ı v)$ todo para hacer obras de provecho $(\chi \rho \eta ́ \sigma u \alpha)$ ” (291d). No obstante, en su tratamiento se toparán con un atolladero: en tanto rige y domina, la técnica real debe tener un objeto, tal como la medicina tiene a la salud. Siendo consecuentes con lo argumentado, ese producto debe ser un conocimiento útil y capaz de volver a los hombres sabios, buenos y felices. Es aquí donde Sócrates indica que llegaron a un límite y no pudieron descubrir respecto de qué la política hace buenos a los hombres. Como se dirá en 293a, ante esta "tercera ola del argumento", pedirán ayuda a los hermanos, quienes lejos de sacarlos del desconcierto, los sumirán en laberintos argumentales tanto o más enrevesados.

La mención a la "tercera ola" es un indicio de la conexión del Eutidemo con República. ${ }^{22}$ Allí esta expresión refiere a la dificultad respecto de la posibilidad de la ciudad ideal, mayor incluso que la relativa a la educación

\footnotetext{
22 Sobre esta cuestión, en la cual no podremos indagar lo suficiente, Cf. Sprague (1976) y Parry (2003).
} 
de las guardianas y a los vínculos familiares de la cúpula gobernante. La fórmula empleada no sólo es la misma sino que su contenido, que los filósofos sean reyes o que los reyes filosofen (Rep. $473 \mathrm{c}-\mathrm{d}$ ), se condice con lo desarrollado en los protrépticos, en tanto liga íntimamente la política con el conocimiento. El contexto de República, con desarrollos ontológicos y epistemológicos robustos que llevarán a aceptar la existencia de Formas y a justificar su inteligibilidad en la postulación de la Forma del Bien (505a), permite ir más allá de la aporía planteada en el Eutidemo, la cual puede considerarse un mero esbozo de la apuesta platónica por el conocimiento

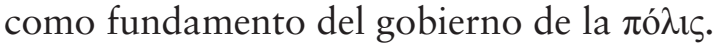

\section{Conclusión}

La crítica final que Sócrates lanza al personaje anónimo constituye un reforzamiento del vínculo entre filosofía y política construido en los protrépticos. Dado lo desarrollado en estas secciones, en el ataque al carácter intermedio y mesurado de este intelectual debería resonar la argumentación que desde el indeterminado deseo de que las cosas nos salgan bien, nos conduce hacia la búsqueda de sabiduría para culminar en la práctica política, enriquecida y guiada, por supuesto, por el conocimiento adquirido en el proceso formativo. Filosofía y política no son en este diálogo actividades diversas que alguien puede combinar a su antojo tomando lo mejor de cada una o, incluso, lo más vistoso a ojos de la mayoría, sino una misma actividad cuyo fin es el bien y la felicidad humana. Será tarea de otros diálogos menos confrontativos, principalmente de República, delinear los contornos exactos de la propuesta filosófico-política de Platón.

Esperamos haber mostrado la doble finalidad que persiguen los dos protrépticos. En tanto contrapunto metodológico a la erística, ambos le permiten a Platón redirigir la crítica de Isócrates a sus condiscípulos más propensos al empleo refutativo de la dialéctica heredada de Sócrates, como Euclides y los megáricos, ya que la manera de emplear dicha forma de argumentación en este diálogo es notoriamente diferente a la de estos: sólo una apariencia externa similar persiste como rasgo común. Desde un punto de vista doctrinal, por su parte, el contenido de los protrépticos es una respuesta contundente a Isócrates y su ridiculización de la pretensión de fundar el ámbito práctico en la sabiduría y el conocimiento. En la reafirmación del saber como lo propio de la filosofía y el consecuente descrédito de la opinión, concepto asociado a la figura del crítico, está quizás la enseñanza más importante que pueda extraerse del Eutidemo. 
Nuestra insistencia en la hipótesis del Eutidemo como respuesta a Isócrates radica en su potencia hermenéutica para dar cuenta de muchas de las tensiones que atraviesan el diálogo, condensadas todas ellas en el epílogo. Para concluir, me gustaría destacar dos de ellas. En primer lugar, el que Sócrates adopte una posición ambivalente respecto de los erísticos ha llamado desde siempre la atención de los especialistas. Lo más llamativo es que nunca reniegue de su cercanía con ellos. A pesar de que podría haber apelado a sus diferencias para sortear la crítica del innominado, Sócrates no duda en colocarse junto a ellos en lo relativo a su dedicación a la filosofía. La mayor ironía de Platón quizás no sea elogiar tan exageradamente a los erísticos, sino negarle a Isócrates el título de legítimo filósofo y hacer de él un crítico poco lúcido de una actividad de la que él mismo se consideraba parte. El epílogo del Eutidemo cierra una obra que debe ser leída más como un llamado de atención a sus condiscípulos desviados que un ataque contra ellos. El tono de advertencia y consejo que impregna la crítica final a la erística contrasta con la subestimación total de una propuesta mesurada como la isocrática.

En segundo lugar, el epílogo condensa la importancia que el concepto de $\varphi \imath \lambda$ iлopía estaba adquiriendo a inicios del siglo IV a. C. y su vínculo con las distintas propuestas teóricas y educativas de la época. Respecto de esto, suele destacarse que fueron Platón e Isócrates quienes tuvieron el rol predominante en la disputa por apropiarse de este término, siendo el primero el que ganaría y sentaría las bases de la filosofía en tanto disciplina autónoma. No obstante, a partir de los discursos de Isócrates analizados y el Eutidemo mismo sería defendible la implicancia de otros intelectuales en tal disputa, como Antístenes y los megáricos, algo que no resulta extraño si tenemos en cuenta que es posible hallar usos importantes del término en la tradición socrática.

Bajo esta hipótesis, la desesperanza final de Critón podría ser fruto de una consciencia en la proliferación de propuestas teóricas y educativas propia de inicios del siglo IV a. C., la cual podía dejar sin un criterio claro a quien buscara dónde hacer pie. El origen compartido de muchas de estas líneas propiciaría aún más el desconcierto. Si el Eutidemo resulta eficaz es porque busca posicionarse en tal clima intelectual, destacando el carácter diferencial y superior de la propuesta platónica frente a las demás. Respecto de la erística megárica, por un lado, porque la suya brinda un andamiaje metodológico para propuestas teóricas positivas, punto en el cual la dialéctica de este grupo se revela incapaz. Respecto de Isócrates, por otro, porque la utilización de la dialéctica como método propio de la filosofía no es ajena 
a planteamientos políticos serios, preanunciando así desarrollos teóricos caros al pensamiento platónico maduro.

\section{Bibliografía}

Canto, M. (1989), Platon. Euthydème. Paris, Flammarion.

Chance, T. (1992). Plato's Euthydemus: Analysis of What Is and Is Not Philosophy. Berkeley-Los Angeles, University of California Press.

Classen, J. (1979). “Aristotle's picture of the sophists”, en G. B. Kerferd (Ed.), The Sophists and Their Legacy. Wiesbaden, Franz Steiner Verlag Gmbh, pp. 7-24.

Classen, J. (1984). "Xenophons Darstellung der Sophistik und der Sophisten". Hermes, 112.2, pp. 154-167.

Clay, D. (1994). “The Origin of the Socratic Dialogue”, en P. V. Waerdt (Ed.), The Socratic Movement. Ithaca, Cornell University Press, pp. 23-47.

Cole, Th. (1991). The Origins of Rhetoric in Ancient Greece. Baltimore, The Johns Hopkins University Press.

Corey, D. (2015). The Sophists in Plato's Dialogues, Albany, State University of New York Press.

Crombie, I. M. (1963). An Examination of Plato's Doctrines. Vol. II. Routledge y Kegan Paul, Londres.

Denyer, N. (1991). Language, Thought and Falsehood in Ancient Greek Philosophy. Londres-New York, Routledge.

Dorion, L. A. (2000). "Euthydème et Dionysodore sont-ils des Mégariques?", en T. Robinson, y L. Brisson (Eds.), Plato. Euthydemus, Lysis, Charmides. Proceedings of the V Symposium Platonicum. Sankt Augustin, Academia Verlag, pp. 35-50.

Dusanic, S. (1999). "Isocrates, the Chian Intellectuals and the Political Context of the Euthydemus". The Journal of Hellenic Studies, 119, pp. 1-16. https://doi.org/10.2307/632309

Gardella, M. (2013). "Conflictos socráticos en el Eutidemo: la crítica platónica a la dialéctica megárica". Argos. Revista de la Asociación Argentina de Estudios Clásicos, 36, pp. 45-64.

Giannantoni, G. (1990). Socratis et socraticorum reliquiae. Nápoles, Bibliopolis.

Gill, C. (2000). "Protreptic and Dialectic in Plato's Euthydemus", en T. Robinson y L. Brisson (Eds.), Plato. Euthydemus, Lysis, Charmides. Proceedings of the V Symposium Platonicum. Sankt Augustin, Academia Verlag, pp. 133-143. 
Grote, G. (1850). History of Greece VIII. Cambridge, Cambridge University Press.

Guthrie, W. K. C. (1969). Historia de la filosofía griega III. Siglo V. Ilustración. Madrid, Gredos.

Hawtrey, R. (1981). Commentary on Plato's Euthydemus. Philadelphia, American Philosophical Society.

Hitchcock, D. (2000). "The origin of professional eristic", en T. Robinson y L. Brisson (Eds.), Plato. Euthydemus, Lysis, Charmides. Proceedings of the V Symposium Platonicum. Sankt Augustin, Academia Verlag, pp. 59-67.

Jebb, R. C. (1876). Attic Orators from Antiphon to Isaeos. Volume 2. Cambridge, Cambridge University Press.

Kerferd, G. B. (1981). The Sophistic Movement. Cambridge, Cambridge University Press.

Livingstone, N. (2007). “Writing Politics: Isocrates' Rhetoric of Philosophy". Rhetorica: A Journal of the History of Rhetoric, 25.1, pp. 15-34. https://doi.org/10.1525/rh.2007.25.1.15

Mársico, C. (2010). Zonas de tensión dialógica. Perspectivas para la enseñanza de la filosofía griega. Buenos Aires, Libros del Zorzal.

Mársico, C. (2014). Filósofos socráticos. Testimonios y fragmentos II: Antístenes, Fedón, Esquines y Simón. Buenos Aires, Losada.

Mársico, C. y H. Inverso (2012). Platón. Eutidemo. Buenos Aires, Losada.

Marcos, G. E. (2000), "Las Falacias en torno a la Falsedad. Una lectura de Eutidemo 283e-286b a la Luz de la Solución del Sofista", en T. Robinson y L. Brisson (Eds.), Plato. Euthydemus, Lysis, Charmides. Proceedings of the V Symposium Platonicum. Sankt Augustin: Academia Verlag, pp. 144-153.

Mathieu, G. y Brémond, E. (1963). Isocrate. Discours I. Paris, Les Belles Letres.

Méridier, L. (1931). Platon: Ion, Ménexène, Euthydème. Paris, Les Belles Lettres.

Mirhady, D. y Y. L. Too (2000). Isocrates. Vol. 1, Austin, University of Texas Press.

Narcy, M. (1984). Le philosophe et son doublé. Un commentaire de l' Euthydème de Platon. Paris, Vrin.

Nightingale, A. W. (1995). Genres in Dialogue. Plato and the Construct of Philosophy. Cambridge, Cambridge University Press. https://doi.org/10.1017/CBO9780511582677

Norlin, G. (1929). Isocrates with and English Translation in Three Volumes. Vol. II. London y New York, William Heinemann y G. P. Putnam's Sons. 
Parry, R. (2003). “The Craft of Ruling in Plato's Euthydemus and Republic". Phronesis 48.1,pp. 1-28. https://doi.org/10.1163/156852803321219761 Rappe, S. (2000). "Father of the Dogs? Tracking the Cynics in Plato's Euthydemus". Classical Philology, 95.3, pp. 282-303. https://doi. org/10.1086/449498

Rossetti, L. (1974). "Alla ricerca dei logoi sokratikoi perduti (I-III)", Rivista di Studi Classici, 22 y 23.

Schiappa, E. (1990). "Did Plato Coin Rhetoriké?”. The American Journal of Philology, 111.4, pp. 457-470. https://doi.org/10.2307/295241

Schiappa, E. (1995). "Isocrates' Philosophia and Contemporary Pragmatism", en S. Mailloux (Ed.), Rhetoric, Sophistry, Pragmatism. Cambridge, Cambridge University Press, pp. 33-60. https://doi.org/10.1017/ CBO9780511597466.002

Sermamoglou-Soulmadi, G. (2014). Playful Philosophy and Serious Sophistry. Berlin-Boston, De Gruyter. https://doi.org/10.1515/9783110365870

Sprague, R. (1972). The Older Sophists. Indianapolis, Hackett Publishing Company.

Sprague, R. K. (1976). Plato's Philosopher-King. Columbia, University of South Carolina Press.

Timmerman, D. (1988). "Isocrates' Competing Conceptualization of Philosophy”. Philosophy \& Rhetoric, 31.2, pp. 145-159.

Usacheva, A. (2013). "Socratics as the Addresses of Isocrates' Epideictic Speeches (Against the Sophists, Encomium of Helen, Busiris)", en F. de Luise y A. Stavru (Eds.), Socratica III. Studies on Socrates, the Socratics, and Ancient Socratic Literature. Sankt Augustin, Academia Verlag, pp. 183-190.

Van Hook, L. (1945). Isocrates with and English Translation in Three Volumes III. Vol. I. Cambrigde, Massachusets y London, Harvard University Press y William Heinemann.

Vigo, A. (2001). "Platón, en torno a las condiciones y la función del diálogo cooperativo". Tópicos, 9, pp. 5-41. https://doi.org/10.14409/topicos. v0i8-9.7404 